\title{
Understanding Curvature Effects on Magnetic Domains in 3D Nanostructures
}

\author{
C. Phatak $^{1}$, C. S. Miller ${ }^{2}$, Z. Thompson ${ }^{3}$, A. Petford-Long ${ }^{1,3}$ \\ ${ }^{1}$ Materials Science Division, Argonne National Laboratory, Lemont, IL 60439, USA \\ ${ }^{2}$ Center for Nanoscale Materials, Argonne National Laboratory, Lemont, IL 60439, USA \\ ${ }^{3}$ Department of Materials Science and Engineering, Northwestern University, 2200 Campus Drive, Evanston, \\ IL 60208, USA
}

The effect of curvilinear geometry and resulting boundary conditions in confined magnetic nanostructures can lead to novel exchange-driven interactions such as effective anisotropy and an antisymmetric vector exchange anisotropy [1]. These novel effects are dependent on the curvature and the curvature-gradient of the nanostructure, and result in magnetochiral and topologically-induced spin textures that have led to theoretical predictions of unlimited domain wall velocities and spin chirality symmetry breaking [2]. In order to understand the interplay between geometric topology and magnetic spin topology, it is critical to understand the magnetic domain configuration and the domain wall behavior in such curved nanostructures at high-resolution and in three-dimensions. Lorentz transmission electron microscopy (LTEM) provides a unique combination to characterize not only the microstructure but also the magnetic domain structure of such nanofibers at a high spatial resolution [3].

In this work, we have fabricated curved magnetic nanohelices using direct focused electron beam ion deposition (FEBID) from Co precursor. This was achieved using a Co precursor gas in the gas injection system on a FEI Nova FIB. We were able to control the growth rate and susbequently the three-dimensional structure of these nanohelices using various electron beam currents and electron accelerating voltage. We will discuss in detail the growth of such nanohelices as shown in Figure 1. The three-dimensional morphology of the nanohelices was studied using electron tomography. We calculated the variation of mean curvature along the helix, which can then be correlated with the resultant magnetic domain structure. The magnetic domain structure was studied using combination of off-axis electron holography and transport-of-intensity formalism. Figure 2 shows the magnetic phase shift, and resultant magnetic induction map from one such helix. We will further discuss the results of correlating the magnetic domain structure with the geometric curvature of the 3D nanohelices, as well as present the three-dimensional magnetization mapping.

References:

[1] Y. Gaididei, V.P. Kravchuk, D.D. Sheka, Phys. Rev. Lett., 112, 257203 (2014).

[2] J. A. Otalora, J.A. Lopez-Lopez, P. Vargas, P. Landeros, Appl. Phys. Lett., 100, 072407 (2012).

[3] C. Phatak, et. al., Nano Lett., 14, 759 (2014).

[4] This work was supported by U.S. Department of Energy (DOE), Office of Science, Office of Basic Energy Sciences, Materials Sciences and Engineering Division.

[5] Use of the Center for Nanoscale Materials, an Office of Science user facility, was supported by the U.S. Department of Energy, Office of Science, Office of Basic Energy Sciences, under Contract No. DEAC02-06CH11357. 


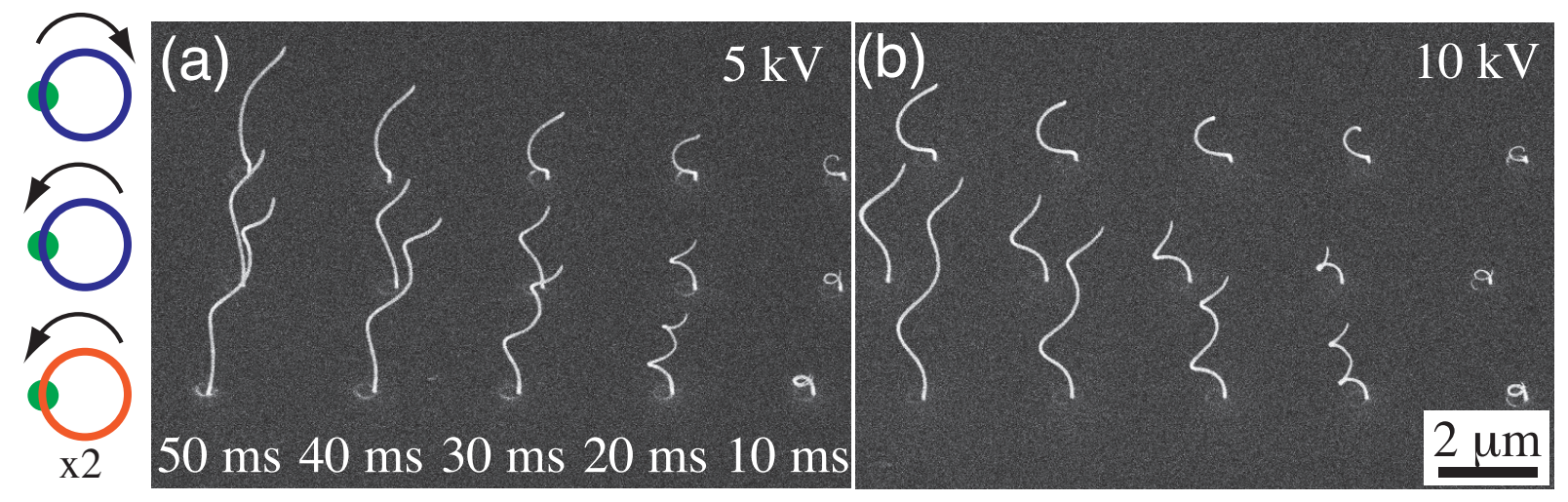

Figure 1: SEM images showing the fabrication of curved 3D cobalt nanohelices using FEBID for (a) $5 \mathrm{kV}$ and (b) $10 \mathrm{kV}$. From left to right, the dwell time per point decreases from $50 \mathrm{~ms}$ to $10 \mathrm{~ms}$. In each image, the top row shows the fabrication of clockwise helix, the middle row shows counter-clockwise, and the bottom row shows two loops counter-clockwise as indicated in the schematic.
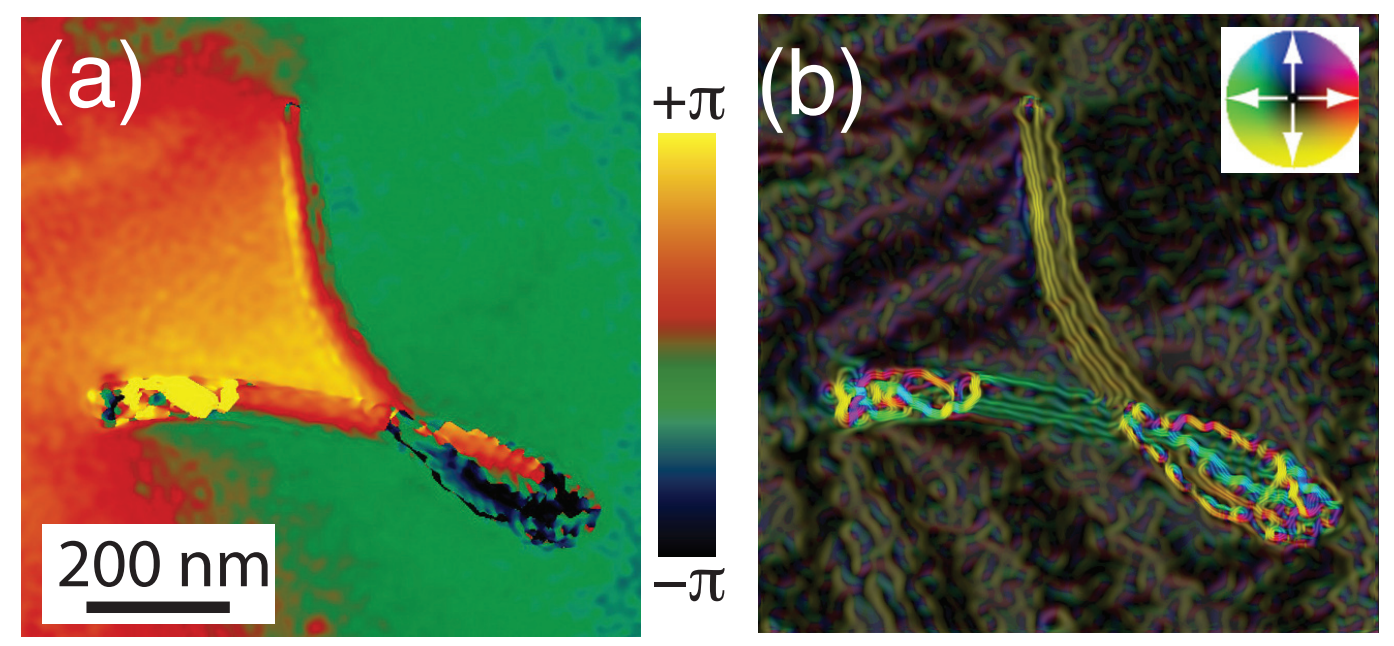

Figure 2: (a) shows the reconstructed magnetic phase shift from a nanohelix where the color indicates the range of phase from $-\pi$ to $+\pi$, and (b) shows the colored magnetic induction map of the helix showing the direction of magnetization as per the inset colorwheel. 